\title{
Performance Analysis of Sub-Rating for Handoff Calls in HCN
}

\author{
Xiaolong WU ${ }^{1}$, Min HE ${ }^{1}$, Fei WANG ${ }^{2}$, Jun ZHENG ${ }^{3}$, Emma REGENTOVA ${ }^{4}$, Guoshun HAO ${ }^{5}$ \\ ${ }^{1}$ Department of CECS, ${ }^{2}$ Department of EE, California State University, Long Beach, CA, USA \\ ${ }^{3}$ Department of Computer Science, New Mexico Institute of Mining and Technology, New Mexico, USA \\ ${ }^{4}$ Department of Electrical and Computer Engineering, University of Nevada, Las Vegas, Nevada, USA \\ ${ }^{5}$ National Lab of Software Development Environment, Beijing University of Aeronautics and Astronautics, Beijing, China \\ Email: $\{x w u 3$, mhe, fwang3\}@csulb.edu \\ Received October 3, 2008; revised December 28, 2008; accepted December 31, 2008
}

\begin{abstract}
Hierarchical Cellular Networks $(\mathrm{HCN})$ offer more efficient channel utilization and better quality of service (QoS) under the high tele-traffic condition compared to the single-tier system. One of the important measures of QoS in $\mathrm{HCN}$ as in any single-tier system is the handoff dropping rate. Although the existing approaches such as guard channel and queuing can reduce forced termination probability, they also result in higher new call blocking probability. The channel sub-rating strategy has found to be an effective technique to reduce the handoff force termination probability while preserving the new call blocking probability in a single-tier system. In this paper, we propose a new call admission control scheme for HCN based on the channel sub-rating. Analytic models based on 1-D Markov process in microcell and 2-D Markov process in macrocell are developed. Experimental results show that our scheme achieves lower blocking and forced termination probabilities compared to the traditional guard channel scheme. The effect of channel sub-rating on the voice quality degradation is also studied. Results demonstrate that we can establish a good balance between the forced termination probability and the voice quality degradation by varying the number of sub-ratable full-rate channels.
\end{abstract}

Keywords: Sub-Rating, HCN, Handoff, Degradation Ratio

\section{Introduction}

Cellular networks has experienced a rapid growth of the number of subscribers during the past decades, which places high demands on the system capacity [1-4]. The cell size can be reduced to accommodate more mobile users in a given area and higher frequency re-uses [5]. However, chances of mutual interferences and cellboundary crossings (handoffs) in small-cell systems are high [6]. The encountered handoffs can degrade one of the essential performance characteristics of cellular networks, i.e., forced termination probability—probability of dropping an active call. Handoff calls limit the call handling capacity of a cellular system due to higher delays and limited bandwidth source. One way of controlling the increase of traffic, while preserving the frequency reuse advantage of small-cell systems is to consider hierarchical cellular network (HCN) [7-8]. In $\mathrm{HCN}$, cells of different sizes are organized into separate layers to provide high coverage and capacity over a given service area. For example, in the two-layer (micro/ macro) hierarchical structure [9], several small cells called microcell with a radii of a few hundred meters are organized as a microcell layer which is overlaid by a large macrocells whose radius is a few kilometers.

In $\mathrm{HCN}$, upon entering the system, the mobile terminals can select the service layer based on their mobility or traffic patterns. For example, in the speedinsensitive selection mechanism [10], all new and handoff calls are first directed to the microcells; if there is no channel available in the microcells, calls can be overflowed to the macrocell. On the contrary, in the speed-sensitive strategy, the mobile terminals traveling at high speeds (fast-mobility subscribers) are normally serviced by the macrocell, whereas low-mobility subscribers are handled by the microcells [4,9-12]. Under the speed-sensitive layer selection strategy, the overflow operation can be used to transfer calls to the other layer if there is no sufficient resource in the current service layer [13-15].

For call admission control (CAC) in HCN, handoff call handling is particularly important because generally the forced termination of an ongoing call is considered 
less desirable than blocking of a new call. Thus, handoff calls have higher priority than new calls during the call admission control process. Several approaches have been proposed for handling handoff calls in HCN. Guard channels $[3,9,16]$ are reserved for serving the handoff calls in both microcells and macrocells. Blocked handoff calls [1] are queued before there is any free channel available to reduce the force termination probability. Lagrange and Goldewski [10] proposed that only handoff calls can overflow from the microcell to the macrocell. Chung and Lee [11] proposed a mobility-dependent call admission control scheme that new calls are accepted according to some mobility-based acceptance probability while handoff calls are admitted when there are free channel available.

These existing schemes can effectively reduce the handoff call forced termination probability, but at the cost of the increment of new call blocking probability. In [5], a new channel assignment scheme called the subrating is introduced to address handoff call problem in the Personal Cellular System (PCS). Sub-rating means that an occupied full-rate channel can be temporarily divided into two channels at the half of the original rate: one to serve the existing call and the other to serve the handoff request. By using the channel sub-rating, it is shown that the forced termination probability is greatly reduced with a slight increase of the blocking probability of new calls. The channel sub-rating scheme was used in [17] to improve the performance of handoff packets for the cellular radio network. The proposed scheme which combines fixed channel assignment, sub-rating, and directed retry, gives a significant improvement in the QoS of the cellular radio network. In [18], Yamanaka and Shimohara applied channel sub-rating for handoff calls in CDMA cellular networks. However, in all of the aforementioned studies, the channel sub-rating is only used in a single-tier system. Even some preliminary results about the utilization of sub-rating in $\mathrm{HCN}$ were reported in [19], this paper gives a complete investigation of the sub-rating strategy for handoff calls in HCN. A general two-tiers HCN system model is considered in this paper. Performance models are developed for the microcell layer and macrocell layers respectively. Two important QoS merits, new call blocking probability and handoff call forced termination probability, are computed to evaluate the proposed call admission control scheme. Meanwhile, the degraded voice quality due to the half-rate channel is also investigated to study the effects of the sub-rating on the QoS. Furthermore, the partial sub-rating by decreasing the number of sub-ratable full-rate channel is taken into consideration to balance the forced termination probabilities and the voice quality.

The rest of the paper is organized as follows. The proposed CAC strategy with channel sub-rating and unidirectional call overflow is introduced in Section 2. In Section 3, the system model and performance analysis of micro/macro layers are described with details. Numerical results are presented in Section 4. Finally, concluding remarks are given in Section 5.

\section{Call Admission Control With Sub-Rating}

The HCN system supports both fast-mobility users and low-mobility users, e.g. pedestrians. We use the speedsensitive selection strategy, i.e., the macrocells handle the fast-mobility users while the microcells serve the low-mobility users. In the following, we describe the proposed CAC scheme with the channel sub-rating. Notice that some other techniques such as queuing [5] are orthogonal to the proposed scheme thus not investigated here.

The scheme is illustrated in Figure 1. In each microcell, slow new or handoff calls will be serviced with a full-rate channel if the total number of calls currently being serviced does not exceed the full capacity of the microcell. Otherwise, handoff requests are handled by using sub-rating that is an occupied full-rate channel but temporarily divided into two channels at a half of the original rate: one to serve the existing call and the other to serve the new incoming slow handoff request. To reduce the blocking probability of slow new calls, blocked slow new calls are allowed to overflow to the overlaying macrocell.

In a macrocell, fast new or handoff calls and overflowed new slow calls will be served with a full-rate channel if the number of calls exiting does not exceed the full capacity of the macrocell. Otherwise, new calls, including fast new calls and overflowed slow new calls will be blocked, while handoff calls including fast handoff calls and slow handoff calls incurred by the overflowed slow new calls are handled based on the sub-rating, similar to those at the microcell layer. The overflow of the fast calls from macrocell to microcell is not allowed because it will incur frequent handoff of the fast calls in the microcell layer due to small size of a microcell. Takeback is also not used as it needs the system to continuously monitor the available resource in the microcell layer [13].

\section{System Model and Performance Analysis}

\subsection{System Model}

For the analysis purpose, we introduce the system model of the two-tier HCN with the following assumptions.

- A macrocell in the macrocell layer is overlaid completely by $N$ microcells in the microcell layer and there is no macrocell-only or microcell-only area.

- Cells in the same layer have the same shape and size.

- Each microcell contains $C_{m}$ channels and each macrocell contains $C_{M}$ channels.

- The fast-mobility calls are first directed to a macrocell, while slow-mobility calls are first directed to a microcell.

- The velocity of mobile terminals is assumed not to change greatly during its call lifetime.

- The total new call arrival to a cluster formed by a macrocell 
and its associated $N$ microcells follows a Poisson process with the mean arrival rate $\lambda_{n}$.

- The portion of slow new calls in total call arrivals to a cluster is $a$.

- The slow/fast handoff call arrival to a given cell at each layer is assumed to be an independent Poisson process with the mean arrival rates $\lambda_{s h}^{m} / \lambda_{f h}^{M}$, respectively.

- The overflowed slow new calls from the microcell layer arrive at the overlaying macrocell in accordance with a Poisson process with the mean rate $\lambda_{s n o}^{m}$; and the slow handoff calls resulting from the overflowed slow new calls arrive at the macrocell in accordance with a Poisson process with the mean rate $\lambda_{s h}^{M}$.

- The call holding time in a macrocell or microcell is exponentially distributed with the mean rate $1 / \mu$.

- The dwell time is the mobile residence time in a given cell. For a slow mobile, its dwell times are exponentially distributed with the mean rate $1 / \gamma_{s}^{m}$ and $1 / \gamma_{s}^{M}$ in the microcell and macrocell respectively. The dwell time of a fast mobile in the macrocell is exponentially distributed with the mean rate $1 / \gamma_{f}^{M}$.

Since the arrival process of the overflowed calls is generally not Poisson distributed, above assumptions are an approximation made for analytic tractability. Therefore, the slow and fast new call arrival rates can be obtained as

$$
\lambda_{s n}^{m}=\frac{1}{N} a \lambda_{n} ; \quad \lambda_{f n}^{M}=(1-a) \lambda_{n}
$$

Also we can obtain the channel holding time of slow calls in a microcell (or macrocell) which is exponentially distributed with the mean rate of $1 / \mu_{s}^{m}=1 /$ $\left(\mu+\gamma_{s}^{m}\right)$ or $1 / \mu_{s}^{M}=1 /\left(\mu+\gamma_{s}^{M}\right)$. In the same manner, we can find that the channel holding time for fast calls in a macrocell is also exponentially distributed with mean $1 / \mu_{f}^{M}=1 /\left(\mu+\gamma_{f}^{M}\right)$.

Based on the system model, we can perform the performance analysis of the proposed call admission control strategy in the two-layer HCN. In the analysis, we assume that all full-rate channels are sub-ratable and the model can be easily modified for partial sub-rating. The analysis is carried out separately for the microcell and macrocell layers.

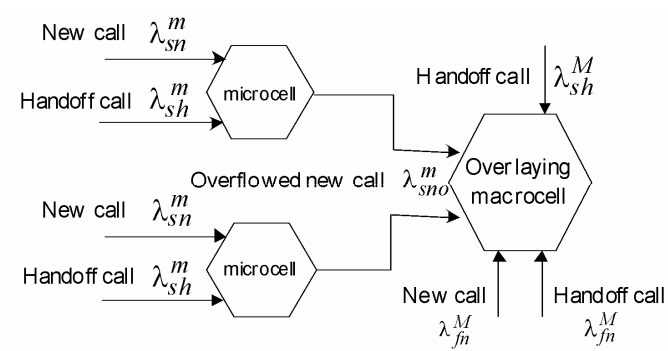

Figure 1. CAC scheme with channel sub-rating and unidirectional calls overflow.

\subsection{Performance Analysis of the Microcell Layer}

Based on the above system model, the microcell layer in the two-layer HCN system can be modeled as a onedimension Markov process.

The state transition diagram of the Markov process is shown in Figure 2. In this diagram, a state is defined as the number of calls in progress in a microcell. For $0 \leq i \leq C_{m}, \quad s(i)$ represents that there are $i$ full-rate busy channels being occupied in a microcell. If there are less than $C_{m}$ busy channels, the arrived slow call (either a new call or a handoff call) is allocated one of those free channels. The Markov process moves from current state $s(i)$ to the next state $s(i+1)$ with the rate $\lambda_{s n}^{m}+\lambda_{s h}^{m}$. The transition rate from state $s(i+1)$ to $s(i)$ is given by $(i+1) \mu_{s}^{m}$.

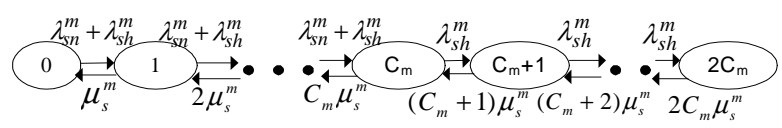

Figure 2. State transition diagram for the microcell.

For $C_{m}<i \leq 2 C_{m}, s(i)$ represents that there are $2 C_{m}-i$ full-rate busy channels, and $2\left(i-C_{m}\right)$ halfrate busy channels. When all full-rate channels in the microcell layer are busy, the arrived slow new call will be blocked while the slow handoff calls will be served by sub-rating a full-rate busy channel into two half-rate channels. If $i=2 C_{m}$, all channels are sub-rated, any arrived slow hand-off call will be blocked. The Markov process moves from $s(i)$ to $s(i+1)$ with the rate $\lambda_{s h}^{m}$. The transition rate from state $s(i+1)$ to $s(i)$ is given by $(i+1) \mu_{s}^{m}$. The steady state probability $\pi_{i}^{m}$ of state $s(i)$ in a microcell is given as follows

$$
\pi_{i}^{m}=\left\{\begin{array}{l}
\frac{\lambda_{s h}^{m}+\lambda_{s h}^{m}}{i ! \mu_{s}^{m}} \pi_{i-1}^{m}, 0 \leq i \leq C_{m} \\
\frac{\lambda_{s h}^{m}}{i ! \mu_{s}^{m}} \pi_{i-1}^{m}, C_{m}<i \leq 2 C_{m}
\end{array}\right.
$$

After the normalization condition that $\sum_{i=0}^{2 C_{m}} \pi_{i}^{m}=1$, it yields

$$
\pi_{0}^{m}=\left[\sum_{i=0}^{C_{m}} \frac{\left(\lambda_{s h}^{m}+\lambda_{s h}^{m}\right)^{i}}{i !\left(\mu_{s}^{m}\right)^{i}}+\sum_{i=C_{m}+1}^{2 C_{m}} \frac{\left(\lambda_{s h}^{m}+\lambda_{s h}^{m}\right)^{C_{m}}\left(\lambda_{s h}^{m}\right)^{i-C_{m}}}{i !\left(\mu_{s}^{m}\right)^{i}}\right]
$$

From Equations (2) and (3), the respective probabilities of the new and handoff slow calls being blocked in a microcell, $P_{s n b}^{m}$ and $P_{s h b}^{m}$, are expressed as

$$
P_{s n b}^{m}=\sum_{i=C_{m+1}}^{2 C_{m}} \pi_{i}^{m} ; \quad P_{s h b}^{m}=\pi_{2 C_{m}}
$$

According to the CAC scheme described in Section 2, the new slow calls that are blocked at the microcell layer 
are not dropped but overflow to the overlaying macrocell (see Figure 2). Based on Equation (3), the aggregate mean arrival rate $\lambda_{\text {sno }}^{m}$ of the overflowed slow new call from $N$ microcells to the overlaying marocell can be determined as

$$
\lambda_{\text {sno }}^{m}=N P_{s n b}^{m} \lambda_{s n}^{m}
$$

\subsection{Performance Analysis of the Macrocell Layer}

Based on the above assumptions, the total call flow to an individual macrocell can be divided into the following four portions: 1) fast new calls with the arrival rate of $\left.\lambda_{f n}^{M} ; 2\right)$ fast handoff calls with the arrival rate of $\lambda_{f h}^{M} ; 3$ ) overflowed slow new calls with the arrival rate of $\left.\lambda_{s n o}^{m} ; 4\right)$ handoff calls resulting from the overflowed slow new calls with the arrival rate of $\lambda_{s h}^{M}$.

The call state process in the marcocell layer can be modeled by a two-dimensional Markov process as shown in Figure 3. For each state $(i, j), i$ represents the number of fast calls including the fast new and handoff calls, and $j$ is the number of slow calls including the overflowed slow new calls and the incurred slow handoff calls in the macrocell layer. The maximum channel capacity of a macrocell is $2 C_{M}$, which means $0 \leq i+j \leq 2 C_{M}$. In the state transition diagram, $\lambda_{f n}^{M}$ and $\lambda_{f h}^{M}$ cause the state

change along the horizontal direction while $\lambda_{s n o}^{m}$ and $\lambda_{s h}^{M}$ alter the state change along the vertical direction.

Following the state transition diagrams in Figure s 3 and 4 , the equilibrium equations can be derived as follows

$$
\begin{aligned}
& \left(\lambda_{f n}^{M}+\lambda_{f h}^{M}+\lambda_{s n o}^{m}+\lambda_{s h}^{M}\right) \pi_{0,0}=\mu_{f}^{M} \pi_{1,0}+\mu_{s}^{M} \pi_{0,1} \\
& \left(\delta_{A} \lambda_{f n}^{M}+\lambda_{f h}^{M}+\lambda_{s n o}^{m}+\lambda_{s h}^{M}+i \mu_{f}^{M}\right) \pi_{i, 0}=(i+1) \mu_{f}^{M} \pi_{i+1,0} \\
& \quad+\mu_{s}^{M} \pi_{i, 1}+\left(\delta_{B} \lambda_{f n}^{M}+\lambda_{f h}^{M}\right) \pi_{i-1,0}, 0<i<2 C_{M} \\
& \lambda_{f h}^{M} \pi_{2 C_{M}-1,0}=2 C_{M} \mu_{f}^{M} \pi_{2 C_{M}, 0} \\
& \left(\lambda_{f n}^{M}+\lambda_{f h}^{M}+\delta_{C} \lambda_{s n o}^{m}+\lambda_{s h}^{M}+j \mu_{s}^{M}\right) \pi_{0, j}=\mu_{f}^{M} \pi_{1, j} \\
& +(j+1) \mu_{s}^{M} \pi_{0, j+1}+\left(\delta_{D} \lambda_{s n o}^{m}+\lambda_{s h}^{M}\right) \pi_{0, j-1}, 0<j<2 C_{M} \\
& \quad \lambda_{s h}^{M} \pi_{0,2 C_{M}-1}=2 C_{M} \mu_{s}^{M} \pi_{0,2 C_{M}} \\
& \left(\lambda_{f n}^{M}+\lambda_{f h}^{M}+\lambda_{s n o}^{m}+\lambda_{s h}^{M}+i \mu_{f}^{M}+j \mu_{s}^{M}\right) \pi_{i, j}= \\
& \quad(i+1) \mu_{f}^{M} \pi_{i+1, j}+(j+1) \mu_{s}^{M} \pi_{i, j+1}+\left(\lambda_{s n o}^{m}+\lambda_{s h}^{M}\right) \pi_{i, j-1} \\
& \quad+\left(\lambda_{f n}^{M}+\lambda_{f h}^{M}\right) \pi_{i-1, j}, 0<i+j<C_{M} \\
& \left(\lambda_{f h}^{M}+\lambda_{s h}^{M}+i \mu_{f}^{M}+j \mu_{s}^{M}\right) \pi_{i, j}= \\
& (i+1) \mu_{f}^{M} \pi_{i+1, j}+(j+1) \mu_{s}^{M} \pi_{i, j+1}+\left(\lambda_{s n o}^{m}+\lambda_{s h}^{M}\right) \pi_{i, j-1} \\
& +\left(\lambda_{f n}^{M}+\lambda_{f h}^{M}\right) \mu_{s}^{M} \pi_{i-1, j}, i+j=C_{M}
\end{aligned}
$$

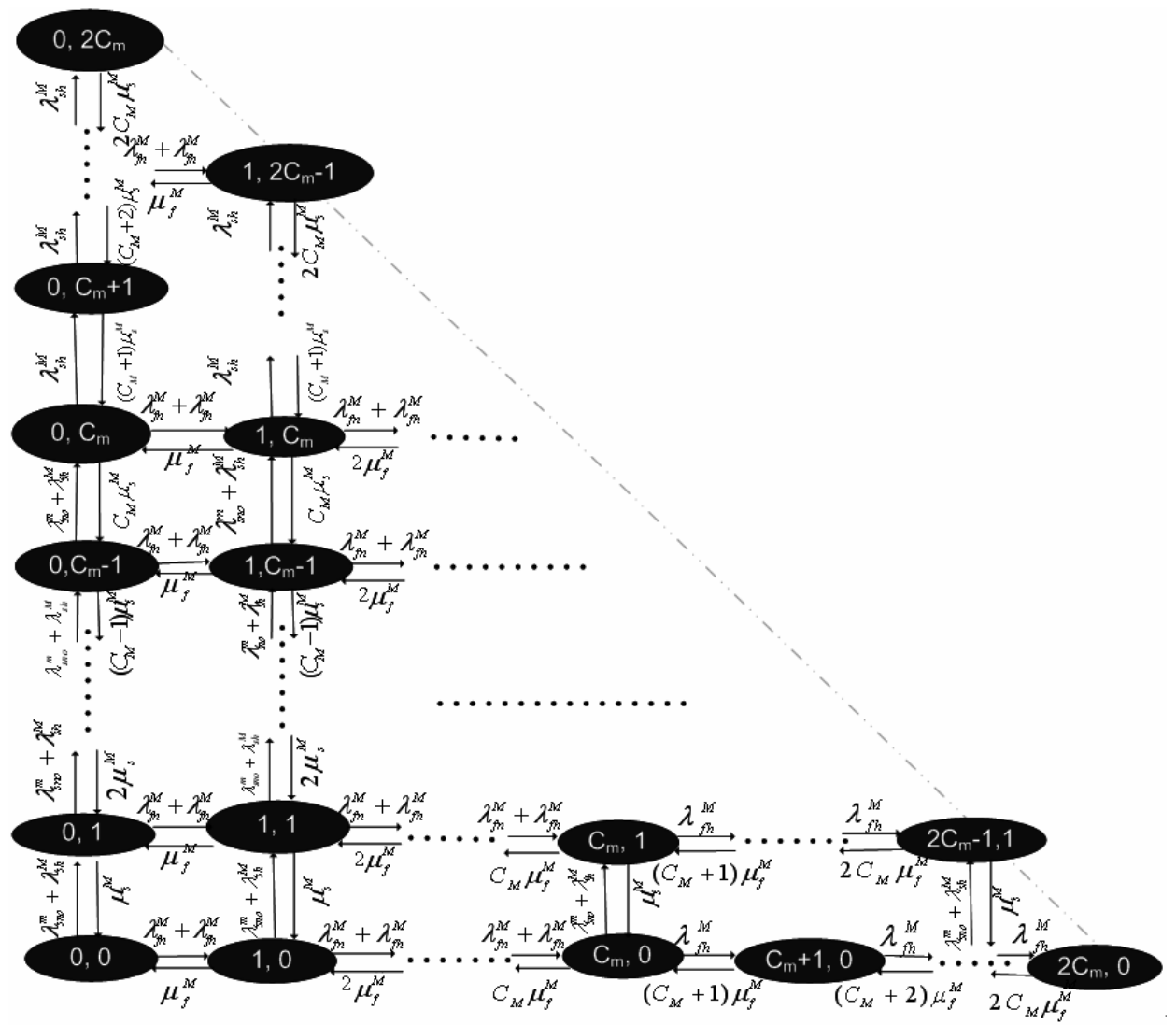

Figure 3. State transition diagram for a macrocell. 


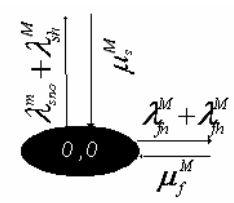

(a) $i=0, j=0$

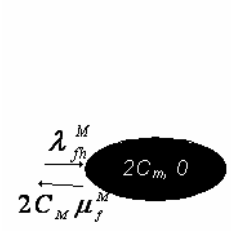

(e) $i=2 C_{M}, j=0$

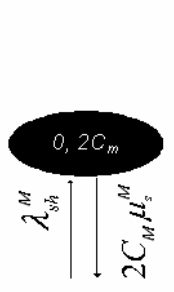

(i) $i=0, j=2 C_{M}$

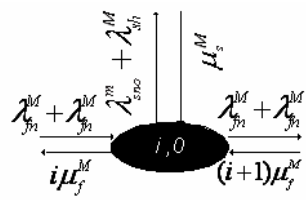

(b) $0<i<C_{M}, j=0$

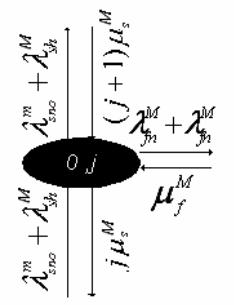

(f) $i=0,0<j<C_{M}$

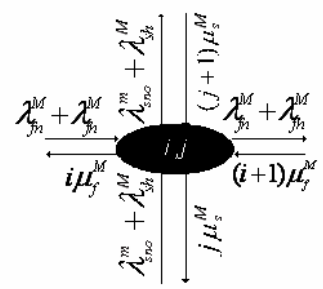

(j) $0<i+j<C_{M}$

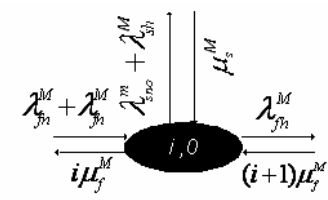

(c) $i=C_{M}, j=0$

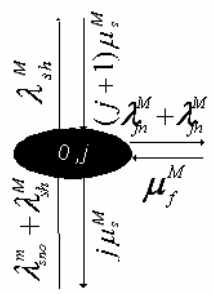

(g) $i=0, j=C_{M}$

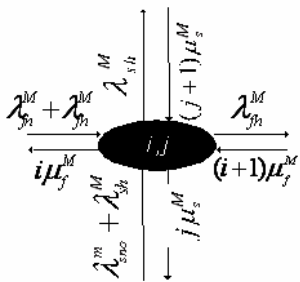

(k) $i+j=C_{M}$

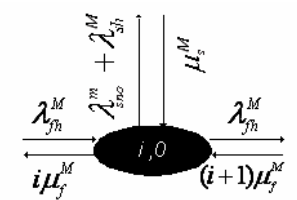

(d) $C_{M}<i<2 C_{M}, j=0$

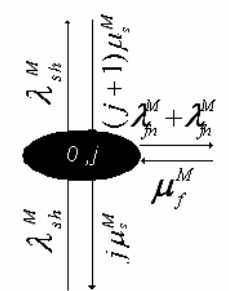

(h) $i=0, C_{M}<j<2 C_{M}$

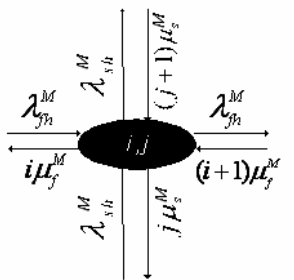

(1) $C_{M}<i+j<2 C_{M}$

Figure 4. Specific states in the state transition diagram.

$$
\begin{aligned}
& \left(\lambda_{f h}^{M}+\lambda_{s h}^{M}+i \mu_{f}^{M}+j \mu_{s}^{M}\right) \pi_{i, j}=(i+1) \mu_{f}^{M} \pi_{i+1, j} \\
& +(j+1) \mu_{s}^{M} \pi_{i, j+1}+\lambda_{s h}^{M} \pi_{i, j-1}+\lambda_{f h}^{M} \pi_{i-1, j}, C_{M}<i+j<2 C_{M}
\end{aligned}
$$

where $\delta_{A}=1$ for $0<i<C_{M}, \quad \delta_{B}=1$ for $0<i \leq C_{M}$, $\delta_{C}=1$ for $0<j<C_{M}$, and $\delta_{D}=1$ for $0<j \leq C_{M}$.

We use the Gauss-Seidel iterative method to solve the above linear equations and compute the steady-state probabilities $\pi_{i, j}$. The probabilities of fast new calls and overflowed slow new calls blocked in the macrocell layer, $P_{f n b}^{M}$ and $P_{s n o}^{M}$, are then expressed as

$$
P_{f n b}^{M}=P_{s n o}^{M}=\sum_{C_{M} \leq i+j \leq 2 C_{M}} \pi_{i, j}^{M}
$$

The blocking probabilities of the fast handoff calls and the overflowed slow handoff calls, $P_{f h b}^{M}$ and $P_{\text {sho }}^{M}$, are expressed as

$$
P_{f h b}^{M}=P_{s h o}^{M}=\sum_{i+j=2 C_{M}} \pi_{i, j}^{M}
$$

From (14) and (15), the total new and handoff slow call blocking probabilities including microcell and macrocell, $P_{s n b}$ and $P_{s h b}$ can be obtained respectively as

$$
P_{s n b}=P_{s n b}^{m} P_{s n o}^{M} ; \quad P_{s h b}=P_{s h b}^{m}+P_{s n b}^{m}\left(1-P_{s n o}^{M}\right) P_{s h o}^{M}
$$

The arrival rates of slow and fast handoff calls can be obtained as in [5] using following three expressions.

$$
\lambda_{s h}^{m}=\frac{\gamma_{s}^{m}\left(1-P_{s h b}^{m}\right)}{\mu+\gamma_{s}^{m} P_{s h b}^{m}} \lambda_{s n}^{m} ; \quad \lambda_{s h}^{M}=\frac{\gamma_{s}^{M}\left(1-P_{s s o}^{M}\right)}{\mu+\gamma_{s}^{M} P_{s h o}^{M}} \lambda_{s n o}^{m} ; \lambda_{f h}^{M}=\frac{\gamma_{f}^{M}\left(1-P_{f n h}^{M}\right)}{\mu+\lambda_{f}^{M} P_{f h b}^{M}} \lambda_{f n}^{M}
$$

Since the handoff rates and the stead state probabilities are mutually related, an iterative algorithm is employed here to compute them.

\subsection{The Degradation Ratio}

The sub-rating scheme is efficient under the limited channel resources because more handoff calls can be served. However, we need to consider the degradation of the voice quality due to the employment of sub-rating. In the following, the degradation ratios of voice quality for the microcell and microcell layers are evaluated.

The expected number $E\left[H_{\text {busy }}^{m}\right]$ of the busy channels in the microcell layer is expressed as

$$
E\left[H_{\text {busy }}^{m}\right]=\sum_{i=0}^{2 C_{m}} i \pi_{i}^{m}
$$

The expected number $E\left[H_{s u b}^{m}\right]$ of the sub-rated channels in the microcell layer is obtained as follows

$$
E\left[H_{s u b}^{m}\right]=\sum_{i=C_{m}+1}^{2 C_{m}} 2\left(i-C_{m}\right) \pi_{i}^{m}
$$

The degradation ratio of the voice quality in the microcell layer, $D R_{m}$, is the portion of the call holding time that the mobile user experiences the degraded voice 
quality [20]. The mean degradation ratio of the voice quality, $E\left[D R_{m}\right]$, can be expressed as

$$
E\left[D R_{m}\right]=E\left[\frac{H_{b u s y}^{m}}{H_{s u b}^{m}}\right]=\sum_{i=C_{m}+1}^{2 C_{m}} \frac{2\left(i-C_{m}\right)}{i} \pi_{i}^{m}
$$

Similarly the mean degradation ratio of the voice quality in the macrocell layer, $\mathrm{E}\left[D R_{M}\right]$, can be obtained as

$$
E\left[D R_{M}\right]=\sum_{(i+j)=C_{M}+1}^{2 C_{M}} \frac{2\left(i+j-C_{M}\right)}{(i+j)} \pi_{i, j}^{M}
$$

\section{Numerical Results}

In this section, numerical results derived from the model developed in Section 3 are presented to quantify the performance of the proposed scheme. The results are compared to the scheme with guard channel and unidirectional call overflow proposed in [9], referred as Shan03 in the plots. If otherwise not specified, the parameters and their values used for the experiments are those shown in Table $1[11,21]$, where $C_{\mathrm{g}}$ and $C_{G}$ are the numbers of guard channels reserved in the microcell and macrocell for Shan03 scheme.

Table 1. Parameters and their values.

\begin{tabular}{cccc}
\hline Parameter & Value & Parameter & Value \\
\hline $1 / \mu$ & $110 \mathrm{~s}$ & $N$ & 4 \\
$1 / \gamma_{s}^{m}$ & $150 \mathrm{~s}$ & $C_{g}$ & 1 \\
$1 / \gamma_{s}^{M}$ & $300 \mathrm{~s}$ & $\mathrm{C}_{\mathrm{G}}$ & 1 \\
$1 / \gamma_{f}^{M}$ & $60 \mathrm{~s}$ & $C_{m}$ & 5 \\
$a$ & 0.8 & $C_{M}$ & 10 \\
\hline
\end{tabular}

\subsection{Case 1: Full Sub-Rating}

In this part, we present the experimental results generated from the model developed in Section 3 where all the full-rate channels are considered to be sub-ratable.

Figure 5 plots the slow/fast new call blocking probabilities and slow/fast handoff call forced termination probabilities under various total new call arrival rates for the proposed scheme and Shan03 Scheme. For Shan03 scheme, we investigate the performance with different guard channel numbers. It can be seen from Figures 5(b) \& (d) that the slow/fast handoff call forced termination probabilities of the proposed scheme are almost zero and also much lower than those of Shan03, even in the case that two guard channels are reserved for handoff calls. Figures 5(a) \& (c) reveal that when the number of guard channel is set to one, the new fast call blocking probability of Shan03 are almost the same as that of the proposed scheme while the new slow call blocking probability of Shan03 is slightly higher than that of the proposed scheme when the number of guard channel is set to two. The reason is that the proposed scheme allows the micro-macro overflow of the new slow calls when

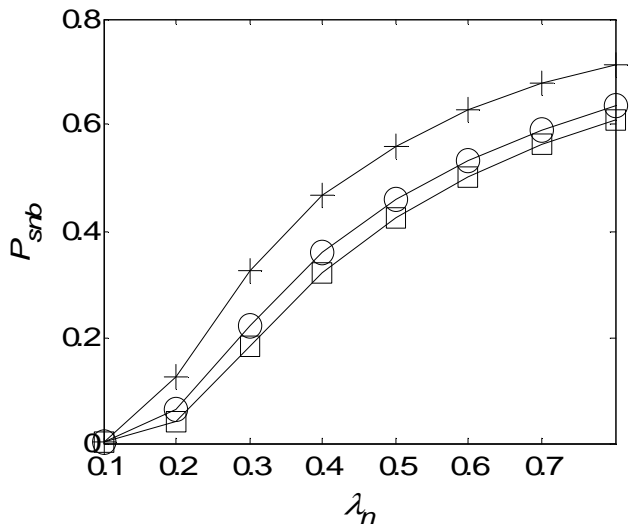

(a)

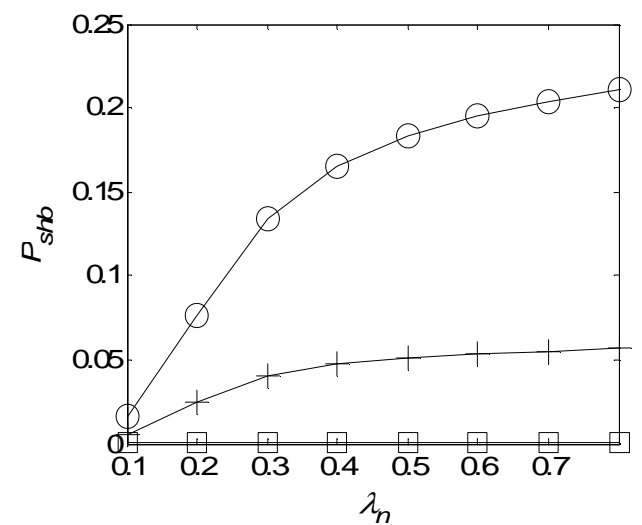

(b)

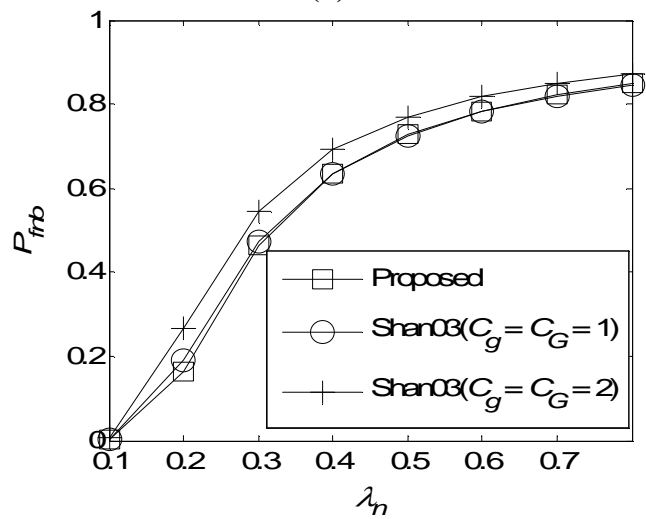

(c)

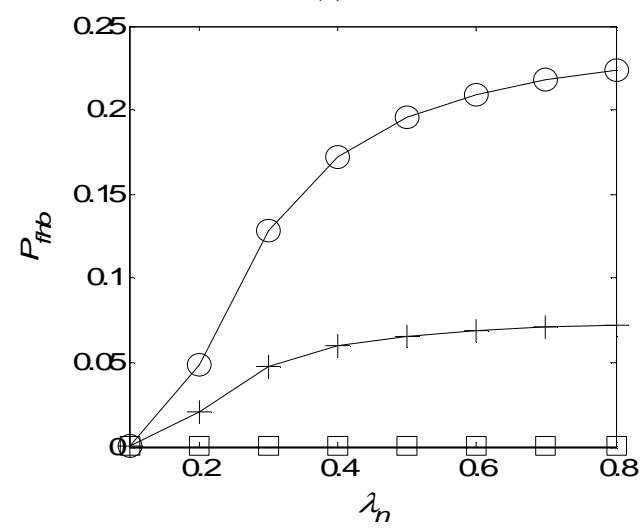

(d)

Figure 5. Performance comparison of the proposed scheme with the Shan03 scheme, $P_{s n b}$, (b) $P_{s h b}$, (c) $P_{f n b}$, (d) $P_{f h b}$. 


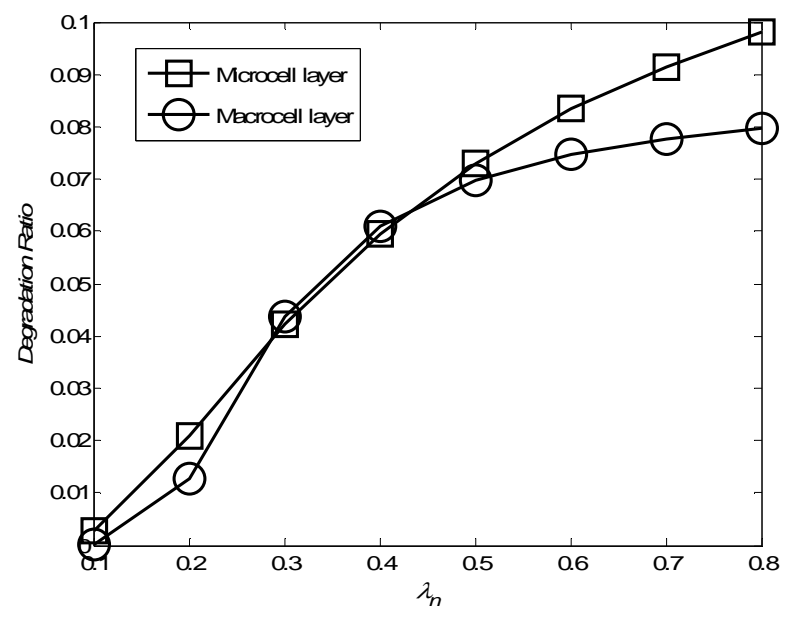

Figure 6. The degradation ratios of the microcell and macrocell layers.

there is no free channel in the microcell. For Shan03 scheme, although reserving more guard channels can greatly reduce the handoff call forced termination probability, the new call blocking probability also increases significantly. The results demonstrate that the proposed scheme achieves better performance for both new and handoff calls than Shan03 scheme in all cases. By employing channel sub-rating strategy, the proposed CAC scheme can massively reduce the probability of forced termination of handoff calls while maintaining the new call blocking probability as low as possible.

Under the channel sub-rating strategy, when calls are in progress, the mobile user is expected to experience the degraded voice quality due to the half-rate channel.

Figure 6 plots the corresponding degradation ratios of the voice quality in the microcell and macrocell layers of Figure 5. Results indicate that the slow and fast mobility subscribers experience the degraded voice quality for a very short period of the total channel holding time when the total new call arrival rate is 0.1 calls/second. As the total new call arrival rate increases, the degradation ratios increase correspondingly. When the total new call arrival rate reaches 0.8 calls/second, the degradation ratios for the microcell and macrocell are about $10 \%$ and $8 \%$, respectively. To reduce the degradation ratio, we can decrease the number of full-rate channel that can be sub-rated which in turn increases the handoff call forced termination probability. Next we will investigate partial sub-rating to balance the forced termination probabilities and the voice quality.

\subsection{Case 2: Partial Sub-Rating}

In this part, we present the experimental results when only partial full-rate channels can be sub-rated. The number of sub-ratable full-rate channels is denoted as $C_{s m} / C_{s M}$ for the microcell/macrocell.

Figure 7 plots the slow and fast handoff call forced termination probabilities and degradation ratios for the microcell and macrocell layers under various $C_{s m}$ and
$C_{s M}$. The total new call arrival rate is set to 0.5 calls/second. It can be seen that the handoff call forced termination probability drops rapidly as the number of sub-ratable full-rate channels increases. On the other hand, the degradation ratios for the microcell and macrocell layers become higher with the increment of the number of sub-ratable full-rate channels. Therefore to meet the QoS requirements of the system on the handoff call forced termination probability and degradation ratio of the voice quality, the number of sub-ratable full-rate channels must be carefully chosen to achieve the best tradeoff.

In Figure 8, we compare the performance of proposed scheme under partial sub-rating with that of Shan03 scheme for various total new call arrival rate, where $C_{s m}$, $C_{s M}, C_{g}$ and $C_{G}$ are both set to 1 . The results demonstrate that the proposed scheme outperforms the guard channelbased Shan03 scheme. Even in this case, only one fullrate channel is allowed to be sub-ratable.

\section{Conclusions}

A call admission control scheme for $\mathrm{HCN}$ has been proposed in this paper which incorporates the channel sub-rating and one-way call overflow. By sub-rating the occupied channels to create new half-rate channels for the handoff calls, the forced terminations of handoff calls are expected to be greatly reduced. Meanwhile, the micro-to-macro overflow was introduced to reduce the blocking probability of slow new calls. We developed analytic models to evaluate the performance of the proposed scheme. Experimental results have shown that the proposed scheme based on channel sub-rating achieves better performance for both new and handoff calls compared to the scheme based on guard channels.
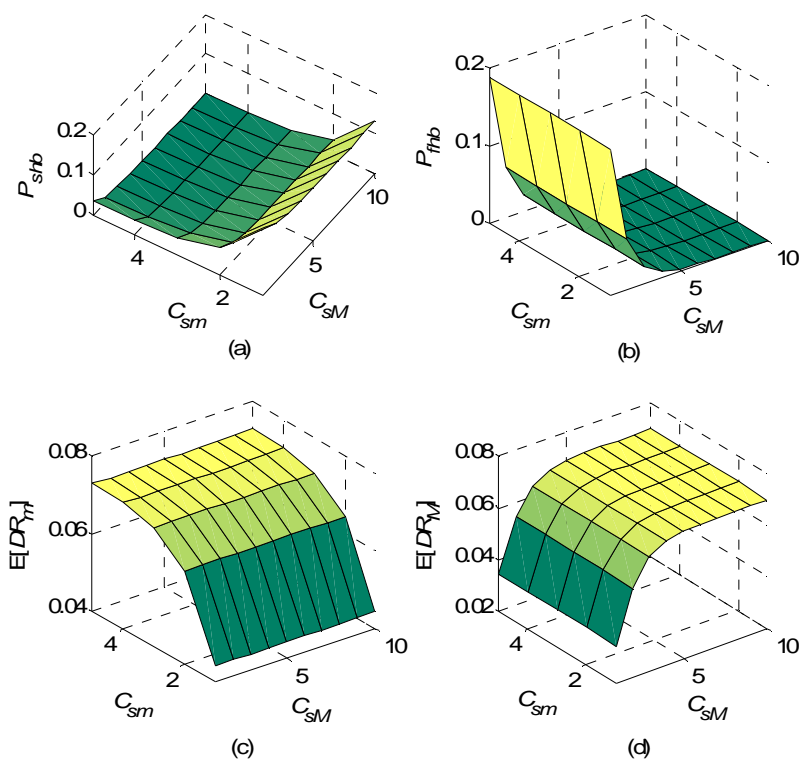

Figure 7. Performance metrics of the proposed scheme under various $C_{s m}$ and $C_{s M}$, (a) $P_{s h b}$, (b) $P_{f h b}$, (c) $\mathrm{E}\left[D R_{m}\right]$, (d) $\mathrm{E}\left[D R_{M}\right]$. 


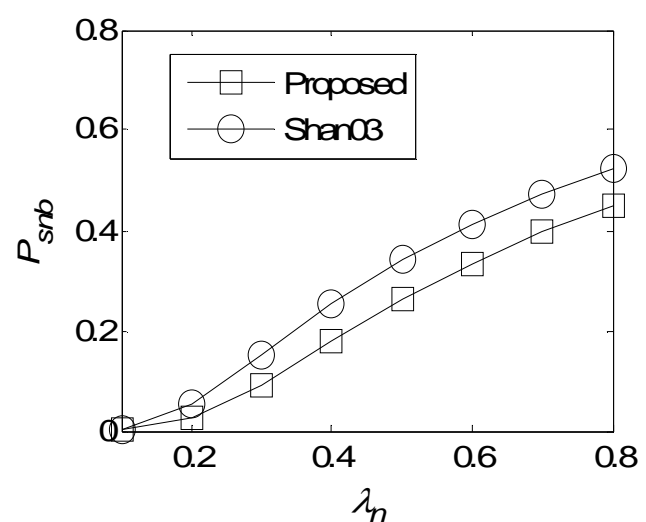

(a)

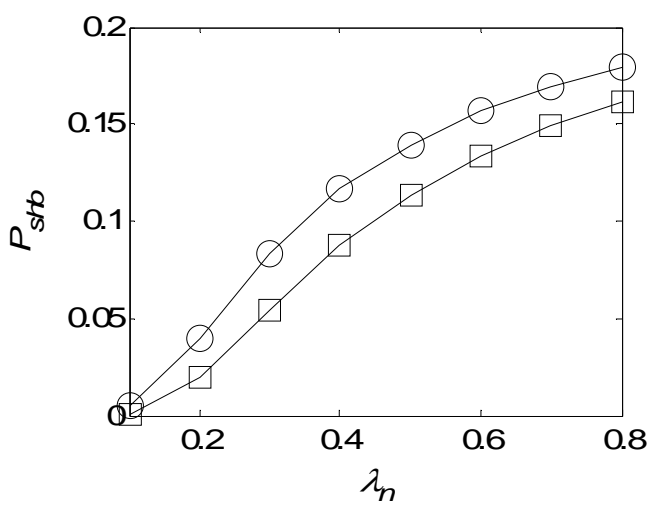

(b)

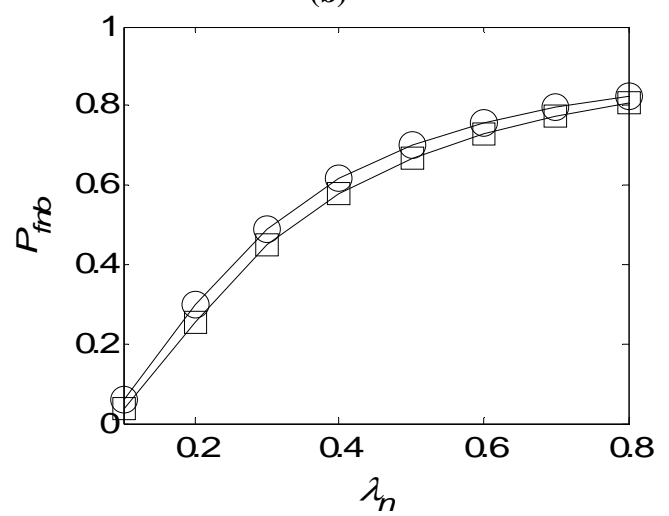

(c)

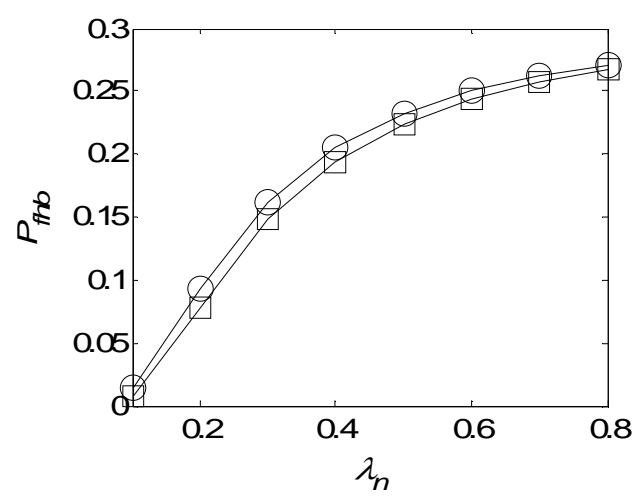

(d)

Figure 8. Performance comparison of the proposed scheme under partial sub-rating with Shan03 scheme for various $\lambda_{n}$, $C_{s m}=C_{s M}=C_{g}=C_{G}=1$, (a) $P_{s n b}$, (b) $P_{s h b}$, (c) $P_{f n b}$, (d) $P_{f h b}$.
The penalty of the sub-rating is the degradation of voice quality during the time when the channels are sub-rated to accommodate the handoff calls. Our results indicated that there is a tradeoff between handoff call forced termination probability and degradation ratio of the voice quality when the channel sub-rating is employed. By assigning the appropriate number of the sub-ratable full-rate channels, we can achieve good balance between the handoff call forced termination and the voice quality degradation.

\section{References}

[1] D. Hong and S. S. Rappaport, "Traffic model and performance analysis for cellular mobile radio telephone systems with prioritized and no-protection handoff procedure," IEEE Transactions on Vehicular Technology, Vol. 35, pp. 77-92, 1986.

[2] Y. S. Hwang, Y. N. Han, and Y. H. Kim, "Performance analysis of mixed voice/data service in a macrocell-based PCS network," IEICE Transactions on Fundamentals, Vol. E81-A, pp. 1136-1144, 1998.

[3] X. Liu and A. O. Fapojuwo, "Performance analysis of hierarchical cellular networks with queuing and user retrials," International Journal of Communication Systems, Vol. 19, pp. 699-721, 2006.

[4] S. S. Tang and W. Li, "Modeling and analysis of hierarchical cellular networks with bidirectional overflow and take-back strategies under generally distributed cell residence times," Telecommunication Systems, Vol. 32, pp. 71-91, 2006.

[5] Y. B. Lin and A. R. Noerpel, "The sub-rating channel assignment strategy for PCS hand-offs," IEEE Transactions on Vehicular Technology, Vol. 45, pp. 122-130, 1996.

[6] K. Maheshwari and A. Kumar, "Performance analysis of microcellization for supporting two mobility classes in cellular wireless networks," IEEE Transactions on Vehicular Technology, Vol. 49, pp. 321-333, 2000.

[7] C. Hartmannn and O. Schelegelmilch, "Hierarchical cell structures with adaptive radio resource management," in Proceedings IEEE Vehicular Technology Conference, pp. 1764-1771, 2000.

[8] X. Yang and M. Guizani, "Optimal paging load balance with total delay constraint in macrocell-microcell hierarchical cellular networks," Vol. 5, No. 8, pp. 22022209, 2006.

[9] W. H. Shan and P. Z. Fan, "Performance evaluation of a hierarchical cellular system with mobile velocity-based bidirectional call-overflow scheme," IEEE Transactions on Parallel and Distributed System, Vol. 14, pp. 72-83, 2003.

[10] X. Lagrange and P. Godlewski, "Teletraffic analysis of a hierarchical cellular network," in Proceedings IEEE Vehicular Technology Conference, pp. 882-886, 1995.

[11] S. Chung and J. Lee, "Mobility-dependent call admission control in hierarchical cellular networks," Computer Communications, Vol. 25, pp. 700-713, 2000. 
[12] S. P. Shung and J. C. Lee, "Mobility-dependent call admission control in hierarchical cellular networks," Computer Communications, Vol. 25, pp. 700-713, 2000.

[13] G. Boggia, P. Camarda, and N. Di Fonzo, "Teletraffic analysis of hierarchical cellular communication networks," IEEE Transactions on Vehicular Technology, Vol. 52, pp. 931-946, 2003.

[14] B. Jabbari and W. F. Fuharmann, "Teletraffic modeling and analysis of flexible hierarchical cellular networks with speed-sensitive handoff strategy," IEEE Journal on Selected Areas in Communications, Vol. 15, pp. 1539$1548,1997$.

[15] Y. Zhang and B. H. Song, "Handoff counting in hierarchial cellular system with overflow scheme," Computer Networks, Vol. 46, pp. 541-554, 2004.

[16] X. Liu and A. O. Fapojuwo, "Resource management for handoff traffic in hierarchical cellular networks," in Proceedings 24th International, Computing, and Communications Conference, pp. 615-620, 2005.
[17] M. J. Rakhee, "A subrating channel assignment scheme for cellular radio network with directed retry," Computers \& Operations Research, Vol. 32, pp. 2407-2417, 2005.

[18] S. Yamanaka and K. Shimohara, "Performance analysis of CDMA cellular networks with channel sub-rating," International Journal of Communication Systems, Vol. 19 , pp. 317-333, 2005.

[19] X. Wu, J. Zheng, E. Regentova, and Y. Jiang, “Analysis of the effect of channel sub-rating in unidirectional call overflow scheme for call admission in hierarchical cellular networks," in Proceedings Vehicular Technology Conference, pp. 1265-1269, 2007.

[20] S. H. Wie, J. S. Jang, B. C. Shin, and D. H. Cho, "Handoff analysis of the hierarchical cellular system," IEEE Transactions on Vehicular Technology, Vol. 49, pp. 2027- 2036, 2000.

[21] S. H. Lee and J. S. Lim, "Performance analysis of channel allocation schemes for supporting multimedia traffic in hierarchical cellular systems," IEICE Trans- actions on Communications, Vol. E86-B, pp. 1274-1285, 2003. 